\title{
Zusätzlich Oxaliplatin bessert Ergebnis bei fortgeschrittenem Rektumkarzinom
}

\section{Die Fluorouracil-basierte präoperative Chemoradiotherapie in Verbindung mit einer postoperativen Chemotherapie ist eine Standardoption beim lokal fortgeschrittenen, zunächst inoperablen Rektumkarzinom. Kann die Addition von Oxaliplatin die Wirksamkeit des Regimes noch einmal verbessern?}

D ieser Frage ging die „German Rectal Cancer Study Group" in der offenen Phase-III-Studie CAO/ARO/AIO-04 nach, in der 1.265 Patienten mit einem rektalen Adenokarzinom des Stadiums cT3-4 oder Lymphknotenmetastasen (cN1-2) randomisiert wurden. Alle Patienten wurden präoperativ mit 50,4 Gy in 28 Fraktionen bestrahlt: Die präoperative Chemotherapie bestand im Standardarm aus $1.000 \mathrm{mg} / \mathrm{m}^{2}$ Fluorouracil (5-FU) i.v. an den Tagen 1-5 und 29-33 und in der Interventionsgruppe aus 5-FU i.v. ( $250 \mathrm{mg} / \mathrm{m}^{2}$, Tage $1-14$ und $22-$ 35) und Oxaliplatin $\left(50 \mathrm{mg} / \mathrm{m}^{2}\right.$, Tage 1,8 ,
22, 29). Nach der mesorektalen Karzinomexzision erhielten die Patienten im Standardarm noch 4 Zyklen mit 5-FUBolusinjektionen an den Tagen 1-5 und 29. Die Patienten in der Interventionsgruppe wurden adjuvant mit 8 Zyklen Oxaliplatin $\left(100 \mathrm{mg} / \mathrm{m}^{2}\right.$, Tage 1,15$)$, Folinsäure $\left(400 \mathrm{mg} / \mathrm{m}^{2}\right.$, Tage 1,15$)$, und 5 -FU $\left(2.400 \mathrm{mg} / \mathrm{m}^{2}\right.$, Tage $\left.1,2,15,16\right)$ behandelt.

Die Addition von Oxaliplatin verbesserte das krankheitsfreie 3-Jahres-Überleben signifikant $(75,9$ vs. $71,2 \%$; Hazard Ratio $\quad 0,79, \quad 95 \%$-Konfidenzintervall $0,64-0,98 ; \mathrm{p}=0,03$ ).
Akute wie auch späte Toxizitäten traten in beiden Patientengruppen in vergleichbarer Größenordnung auf.

Fazit: Oxaliplatin, wenn es beim lokal fortgeschrittenen Rektumkarzinom zusätzlich zu einer präoperativen 5-FUbasierten Chemoradiotherapie und einer 5-FU-basierten postoperativen Chemotherapie gegeben wird, verlängert das krankheitsfreie Überleben solcher Patienten, die sich in einem zunächst nicht operablen Stadium befinden. Möglicherweise könnte eine kombinierte ChemoInduktionstherapie vor der Chemoradiotherapie noch effizienter sein als die postoperative Chemotherapie.

Barbara Kreutzkamp

Rödel C et al. Oxaliplatin added to fluorouracil-based preoperative chemoradiotherapy and postoperative chemotherapy of locally advanced rectal cancer (the German CAO/ARO/ AIO-04 study): final results of the multicentre, open-label, randomised, phase 3 trial. Lancet Oncol. 2015;16(8):979-89.

\section{Hier steht eine Anzeige.}

\section{Springer}

